\title{
Gli scelestissimi lettori di Dante
}

\section{Ricezioni e significati dell'epistola VI nella prima metà del Trecento}

\begin{abstract}
In questo saggio si propone una lettura dell'epistola ai fiorentini del 31 marzo 1311 nella prospettiva dell'orizzonte di ricezione dei destinatari, cioè della comunità dei Guelfi di Firenze nella prima metà del Trecento. Nella prima parte, si tenta di definire attraverso quale paradigma interpretativo un messaggio critico come quello contenuto nella missiva potesse essere filtrato, in relazione ai modi complessivi con cui venne recepita l'opera dantesca. Nella seconda parte invece si cerca di definire con quali pratiche politiche, proprie dei Guelfi fiorentini di inizio secolo, potesse risuonare l'accusa di cupidigia, ovvero l'argomento portante delle critiche espresse dall'autore.

The essay provides a new reading of Dante's epistle to the Florentines (March 31, 1311) in the light of its reception, that is to say in the perspective of its addressees, the Florentine Guelph community of the first half of XIV century. In the first part, I define the reading frame within which such an enigmatic message as that of the epistle could have been interpreted, with respect to the global strategies of reception of Dante's whole production. In the second part I outline which political practices assumed by Florentine Guelphs from the time could be echoed by Dante's condemnation of greed, which is one of the main criticisms expressed by the author.
\end{abstract}

Parole chiave: Dante Alighieri, Epistola VI, Firenze, Angioini, Giovanni Villani.

Oggi la missiva che Dante indirizzò il 31 marzo 1311 agli «scelleratissimi fiorentini intrinseci», ${ }^{1}$ interni cioè alle mura guelfe di Firenze, costituisce il sesto capitolo di un'opera assemblata a metà XIX secolo: le Epistole, ultime arrivate nel catalogo dantesco della modernità a stampa, disponibili dal 1842 in poi. ${ }^{2}$ Anche

1 Ep. (Baglio), VI (le traduzioni sono sempre mie). Sull'edizione, cfr. Montefusco, Epistole a $c$. di M. Baglio.

2 Una descrizione essenziale della tradizione in Ep. (Baglio), Nota ai testi, pp. 29-31 (per la struttura del manoscritto vaticano, cfr. l'articolo di Emanuele Romanini in questo volume).

Amedeo De Vincentiis, Università degli Studi della Tuscia

Ә Open Access. (C) 2020 Amedeo De Vincentiis, published by De Gruyter. (c) BY This work is licensed under the Creative Commons Attribution 4.0 International License (CC BY 4.0). 
se l'anacronismo editoriale rimane corrente, tuttavia la critica filologica ha fornito strumenti e conoscenze utili a smontarlo. Siamo così nelle condizioni di sfilare la testimonianza dalla sua tradizione, dalla serie artefatta dell'epistolario del poeta della Commedia, per rileggerla come un documento a sé stante, ricollocandolo in un contesto tagliato sulla misura storica del suo messaggio.

Se iniziamo a riconsiderare la testimonianza nell'orizzonte del mittente, la lettera agli intrinseci fiorentini svela un tratto d'eccezione, pressoché unico tra gli scritti di Dante. Assieme ad altre due epistole condivide infatti il privilegio di riportare la sola data precisa e inequivocabile segnata su un'opera del fiorentino. ${ }^{3}$ La dimensione storica di riferimento per conseguenza è iscritta nel testo. $\mathrm{E}$ rimane ineliminabile per una decifrazione opportuna, come un codice esegetico imposto dall'autore e vigente dalla prima circolazione.

Proprio per questo durante i primi decenni del XIV secolo la ricezione del messaggio agli scelestissimi, a Firenze, fu condizionata da un dispositivo di interpretazione adattato alla tradizione politica locale. Non tutto ciò che si conosceva dell'opera di Alighieri nel comune guelfo era letto allo stesso modo: attorno ad argomenti speciali, il filtro del dispositivo esercitava resistenze che incoraggiavano la dimenticanza, se non il rifiuto. L'ambiente interpretativo e i confini della decifrazione del guelfismo locale, quindi, furono a lungo parte attiva nel determinare i significati della testimonianza dantesca del 1311 (e probabilmente anche del suo oblio testuale). Su tutto ciò ci soffermeremo nel primo paragrafo, mentre nell'ultimo tenteremo di identificare le dinamiche politiche che costituirono lo sfondo di ricezione delle critiche del poeta, per lo meno all'interno della comunità guelfa. In definitiva, sarà nell'intreccio tra propositi dichiarati dell'autore e attribuzione dei possibili significati da parte degli scelestissimi lettori di Firenze che ritroveremo le potenzialità comunicative del messaggio di Dante.

\section{Nelle mura: il dispositivo di ricezione guelfo}

Nella Firenze di seconda metà del Trecento venne allestito un dispositivo di lettura interpretativa che riammetteva opera e memoria del poeta esule nelle mura del guelfismo di tradizione locale. Attraverso la Nuova cronica di Giovanni Villani, il sapere storico insegnava ai cittadini come i messaggi più critici che

Sulle epistole come pezzi singoli, cfr. Mazzucchi, Introduzione, pp. $\mathrm{xx}$-xxi. Inquadramento della serie in Montefusco, Le "Epistole".

3 Cfr. Santagata, Dante, pp. 415-416. 
Alighieri aveva rivolto loro fossero strumenti di analisi e autocritica politica legittimi, utili alla comunità guelfa, che loro stessi potevano reimpiegare meglio di qualsiasi altro lettore. ${ }^{4}$ Consultando poi il Trattatello di Giovanni Boccaccio, la memoria biografica più autorevole confermava il paradigma, applicandolo alla vicenda civile del poeta. ${ }^{5}$ Ormai Dante Alighieri poteva passare quale sommo moralista, indimenticabile per aver esortato di continuo i concittadini a essere sempre più consapevoli delle debolezze proprie e della comunità. Ciononostante, l'accettazione da parte dei fiorentini di governo manteneva attiva una protezione, invalicabile, a tutela dell'onore del guelfismo locale. Tale filtro si applicava in particolare a tematiche e forme espressive che il messaggio del 1311 concentra forse quanto nessun altro scritto epistolare di Dante. Per questo, ora, è necessario definirlo con attenzione.

Il fiorentino Alighieri mai aveva comunicato con la patria a nome o al servizio di qualche altro potentato - si leggeva nella storia cittadina, come pure nella biografia dell'autore; viceversa, aveva consigliato i residenti nelle mura sempre spontaneamente, per renderli più accorti. Soprattutto, neppure i contenuti delle sue esortazioni poetiche - così come veniva suggerito di interpretarle - recavano accuse mortali contro il guelfismo fiorentino di lunga durata, quello che poggiava sulla rivoluzione degli anni '60 del XIII secolo, quando grazie al favore papale e alle armi franco-angioine la comunità era ridiventata guelfa e quanto mai ortodossa. Insieme al poeta e tanti altri si poteva certo rimpiangere un buon tempo antico e migliore. ${ }^{6}$ Ma era finito agli inizi del Duecento: travolto dall'istituzione imperiale fuori controllo, minacciosamente impiantata nel sud della penisola e pronta a rivendicare la sovranità nel regnum d'Italia da Roma in su. ${ }^{7}$ I mali delle divisioni interne sicuramente si erano esacerbati da allora, e su quelli vita e invettive di Alighieri fungevano da memento esemplare.

4 I passi in Villani, Nuova cronica, X 136; XIII, 19, 97. I dati certi sulla prima circolazione delle opere di Dante sono in Inglese, Vita di Dante, in part. pp. 128-ss. Cfr. Malato, Il mito e, per Firenze, Bellomo, L'interpretazione, in part. pp. 140-141 (e sul filone dei commenti, almeno Bellomo, Dizionario). Per quegli ambiti di circolazione, Steinberg, Dante e il suo pubblico; e anche Ceccherini, Mercanti copisti.

5 Boccaccio, Trattatello. Importanti le messe in prospettiva di G. Billanovich, La leggenda, da integrare con Indizio, Problemi, pp. 263-340 (e più in generale sulle biografie dantesche, pp. 127-172). Per il testo, messa a punto filologica in Bertè, Fiorilla, Il Trattatello, pp. 41-ss. Cfr. Azzetta, Le "Esposizioni”. Sulle biografie dantesche, cfr. la messa a punto di Bartuschat, Le biografie di Dante, pp. 171-ss

6 Rilettura delle testimonianze circa il buon tempo antico in Zabbia, Dalla propaganda, pp. 247-ss.

7 Cfr. Dessì, I nomi. Si veda anche Cherubini, L’immagine; Capo, La cronachistica; Zabbia, Il Regno. 
Inquadrandoli in tale paradigma veniva attenuata l'originalità corrosiva dei messaggi critici dell'esule. Nell'idioma del guelfismo locale, infatti, denunciare conflitti e divisioni dentro le mura appariva più che lecito, corrispondeva persino a un segno di saggezza. E i cittadini che reggevano il comune, quando componevano storie per proprio conto, annotavano memorie o ragionavano di politica nelle mura, esibivano sottili capacità di analisi circa i difetti di funzionamento dei meccanismi sociali e delle pratiche politiche di cui essi stessi erano partecipi. ${ }^{8}$ Quando però il regime agiva verso l'esterno, con voce ufficiale e atti formalmente legittimi, la solidarietà doveva rimanere intatta anche nel ricordo. Anche guardando al passato la soglia dell'accoglienza lecita nel guelfismo più convinto rimaneva fissata sulle conseguenze esterne. Il discrimine dell'accettazione e delle critiche era calibrato sulla messa in discussione del patrimonio materiale, del prestigio di onore e della autonomia dei fiorentini, sia nel dominio regionale che in relazione a qualsiasi altro potentato. ${ }^{9}$ La solidità dell'identità politica del guelfismo locale nella lunga durata, a Firenze, richiedeva di essere garantita degli sguardi esterni. ${ }^{10}$

$\mathrm{Su}$ questo versante, la ricezione guelfa dell'eredità dantesca si mantenne sorvegliatissima. Se nei testi composti in lingua madre la comunanza identitaria con i fiorentini di dentro poteva passare per scontata, molto più resistenti al filtro di rilettura locale rimasero gli scritti latini in cui l'autore aveva esposto la sua visione ideologica e politica. Quei testi infatti si presentavano nelle forme espressive e linguistiche appropriate a saperi con cui erano sostenute verità, argomenti, ragioni, universalmente validi e riconosciuti. Nel loro caso, un lasciapassare postumo nelle mura del guelfismo locale richiedeva molte più cautele.

In quella materia, l'eredità più vistosa rimaneva la Monarchia. Non a caso negli anni '30 tra i Guelfi di Firenze il trattato era ricordato in termini neutri, come una sapiente disquisizione sull'ufficio imperiale. Ancora più prudentemente, nella divulgazione sintetica affidata dal comune al banditore ufficiale Antonio Pucci, al soggetto imperiale si faceva precedere quello pontificio («Dante fece ancor quella Monarchia, / dove de' Papi e dello Imperiato / trattò con molta gran filosofia»). ${ }^{11} \mathrm{E}$ quando il biografo Boccaccio volle presentare il testo più nel dettaglio si dilungò per giustificarne la compatibilità con gli orientamen-

8 Cfr. Arnaldi, Dino.

9 Cfr. De Vincentiis, Origini, memoria, identità, pp. 385-ss. (sulla tradizione fiorentina circa le divisioni, cfr. Pedullà, Machiavelli in tumulto).

10 Trexler, Public.

11 Delle poesie di Antonio Pucci, 55, 77. Cfr. Cella, Il "Centiloquio", e per il ruolo pubblico Robins, Antonio Pucci. La Nuova cronica riporta: «fece ancora la Monarchia, ove trattò de l'oficio degli 'mperadori» (Villani Nuova cronica X, 136). 
ti guelfi. Se era stato censurato dalle autorità ecclesiastiche pontificie fu solo colpa di un uso distorto del trattato, indipendente dalle volontà di Dante - si leggeva nel Trattatello ${ }^{12}$-, nonché dell'accanimento postumo di un malevolo cardinale.

Fin dal suo apparire, e anche in seguito, inoltre, il trattato imperialista di Dante interferì solo in modo obliquo con l'identità del guelfismo locale. Senza prendere di mira specifiche scelte politiche dei regimi dentro le mura, l'opera proponeva piuttosto un modello di ordinamento valido su scala universale, ambientato in una temporalità senza scadenze. Diverso era il caso di un messaggio politico puntuale, stilato in un linguaggio e in codici altrettanto densi di sapere, ma rivolto direttamente alla comunità dei fiorentini che reggevano la città a una data precisa: 31 marzo 1311. La forma stessa impediva l'assimilazione in serie. In questo caso, senza possibili equivoci, l'esperto riconosciuto nelle arti delle scritture comunicative aveva prescelto un codice che mimava gli scambi tra autorità pubbliche, con cui si esprimevano i discorsi alti sugli affari di stato e le analisi ragionate delle situazioni in campo. ${ }^{13}$ La missiva del 1311 dunque era un'interlocuzione che non lasciava lo scampo garantito da altre forme di comunicazione letteraria. Conviene ora rileggerla brevemente in questa prospettiva.

L'epistola Eterni pia providentia fin dall'incipit accordato al tono delle scritture sacre sollecitava un doppio livello di decifrazione. Il messaggio politico riguardava la contingenza della primavera del 1311, e si concludeva con la richiesta di un intervento pragmatico che sarebbe spettato al regime allora in carica: la spontanea accoglienza di Enrico VII di Lussemburgo nel suo passaggio per la città guelfa, in vista dell'incoronazione a Roma. Con altrettanta enfasi, però, nel testo si ambiva ad aprire un canale comunicativo fondato sul riconoscimento delle qualità culturali e intellettuali dei destinatari. Se i lettori moderni rimangono colpiti soprattutto dalla virulenza dei toni e delle espressioni rivolte contro gli scelestissimi fiorentini, i codici retorici impiegati nel messaggio epistolare invece definivano interlocutori considerati particolarmente competenti dall'autore: in grado di apprezzarne lo sforzo compositivo, il pregio delle forme linguistiche, l'arte di modulare i colori retorici e quella di alternare registri discorsivi propri di vari saperi. ${ }^{14}$

Ridotta in sintesi, la logica del messaggio politico dell'esule contrapponeva due sistemi di organizzazione dei poteri nella penisola italica, presentati come

12 Boccaccio, Trattatello, 26. Sul brano, cfr. Casadei, Dante oltre, pp. 124-126.

13 Cfr. la descrizione della forma della scrittura di Dante in Leonardo Bruni, Dialogi, 44; cfr. Ep. (Baglio), pp. 233-234.

14 Sui saperi sacri e filosofici cfr. Brilli, Reminiscenze; Maspoli Genetelli, Filosofia politica. Sul contesto del 1311 cfr. Santagata, Dante, pp. 231-ss. 
inampalibili. Con il linguaggio alto del diritto, innanzitutto si ribadiva che l'opzione di una circoscrizione italica coordinata dall'autorità universale dell'Impero rimaneva del tutto legittima, esente dal «diritto di prescrizione», ${ }^{15}$ e praticabile anche nei tempi attuali. Quindi si condensava la tradizione politica imperiale nella nozione di "utile di tutti", un principio di matrice giuridica ma capace di risuonare in una ampia gamma di linguaggi concettuali. ${ }^{16}$ Infine si prospettava la situazione attuale, sul campo italico di quelle settimane, dominata dalla determinazione dell'imperatore a punire i cittadini nelle mura in caso di opposizione. L'antitesi invece era incarnata dalle convinzioni degli scelestissimi, personificazioni delle ragioni di coloro che sostenevano il sistema di coordinamento guelfo, allora retto dall'amicizia con i sovrani di Napoli. «Vos autem» si rivolgeva diretto l'oratore nel discorso epistolare - avete abbandonato "ciò che reca utilità a tutti» a vantaggio di un diverso e recente ordine politico: cedendo cioè alla tentazione di «nuovi regni» (Ep. VI, 5-8).

Sebbene ritmata da un fraseggio aggressivo, nel messaggio tuttavia la retorica dell'errore primeggiava su quella della colpa. Quando si passava alla politica condotta dagli scelestissimi, infatti, l'argomentazione si fondava su una presupposta cecità politica dei destinatari, che alimentava illusioni sincere, sebbene fallaci. Loro stimavano realmente - «existimatis» rinfacciava Dante (Ep. VI, 13) - di essere protetti da un manto di libertas grazie ai «nova regna» cui si erano rivolti. A considerarla dall'esterno, da fuori le mura, invece, quella appariva una «toga di falsa libertà», ${ }^{17}$ e ciò che l'animava avrebbe precipitato i suoi fedeli nell' «ergastolo di una vera servitù». Preferendo una argomentazione nei modi della confutazione di un inganno intellettuale a quelli giudiziari che avrebbero motivato una sanzione definitiva, senza scampo la strategia comunicativa del messaggio apriva una breccia alla conversione in estremo. In conclusione, una punizione terribile era annunciata per chi si fosse pentito troppo tardi (dopo l'inevitabile vittoria imperiale sotto le mura): il che sottintendeva pure un trattamento diverso per quelli che si fossero subito conformati alla valutazione offerta dall'esule.

In definitiva, il messaggio epistolare appariva costruito per interlocutori in grado di valutarlo sollevandosi dallo spazio stretto dalla cerchia muraria, offrendo loro materia di riflessione su una scala più ampia della contingenza im-

15 «Atque iure prescriptionis utentes» (Ep. VI, 5).

16 «Quod ad omnium cedit utilitatem»; «nova regna temptatis» (Ep. VI, 7-8). Cfr. Hibst, Utilitas; e, in riferimento a Dante e il diritto imperiale, cfr. Quaglioni, Introduzione, pp. lxxii-lxxix (con discussione della bibliografia).

17 «Et quo false libertatis trabeam tueri existimatis eo vere servitutis in ergastula concidetis» (Ep. VI, 13). 
mediata di quelle settimane. A essere messo in questione era il sistema sovraregionale su cui gli scelestissimi avevano puntato le loro fortune civili e politiche. E attraverso l'offerta di una varietà di saperi in cui declinare il tema di fondo dalla teologia, al diritto, alle valutazioni sulle attuali condizioni civili di Firenze, ai precedenti della storia dei tempi di Federico I e Federico II - l'Eterni pia providentia apriva ai destinatari altrettante possibilità di discutere e riflettere su un sistema di alleanze che in quel momento la presenza imperiale permetteva di allentare. Questo avrebbe consentito sostenere Enrico Cesare: stabilire cioè un equilibrio, nell' «Italia misera, sola, lasciata agli arbitri privati», ${ }^{18}$ tra la recente monarchia angioina di Napoli, figlia prediletta del papato di Avignone, cugina dei prepotenti re di Francia, e le comunità della antica circoscrizione del regno italico.

In seguito, fino agli anni '40 del secolo, l'amicizia politica con la dinastia angioina di Napoli rimase un punto fermo per i regimi guelfi che governarono a Firenze. Cosicché anche quando la comunità cittadina avviò una politica culturale di riassorbimento dell'opera dell'esule Alighieri - secondo l'impostazione che abbiamo ripercorso in precedenza -, il messaggio dell'epistola rimase irricevibile nell'orizzonte ideologico dominante. Un conto erano le critiche alla casa di Francia e al ramo angioino sparse nel grande poema volgare, che bersagliavano principi per lo più defunti e, in ogni caso, non li associavano esplicitamente ai fiorentini nelle mura. Altro peso conservava invece la denuncia del legame particolare tra i gruppi dirigenti del comune e i discendenti di Carlo I d'Angiò, tanto più che Dante non si era limitato a criticare un errore di valutazione momentaneo. Nella lettera, in effetti, si forniva una spiegazione approfondita del sistema di interessi che sorreggeva quella opzione politica. Gli scelestissimi continuavano a non vedere - «accecati», "perché siete ciechi», ${ }^{19}$ si leggeva nell'epistola - che solo la presenza del contrappeso imperiale nella penisola poteva garantire una autentica libertà, anche per Firenze, a causa di una pulsione dominante e persuasiva: la cupidigia.

\section{L’avarizia dei nuovi re e la cupidigia degli scelestissimi}

I lettori fiorentini più esperti dell'opera dantesca, incontrando il termine cupiditas nella missiva del 1311, potevano ricordare l'equivalente in lingua madre che

18 «Quod Ytalia misera, sola, privatis arbitriis derelicta» (Ep. VI, 3).

19 «Obcecati» (Ep. VI, 12); «nec advertitis dominantem cupidinem, quia ceci estis» (Ep. VI, 22). 
ricorreva altrove negli scritti del concittadino. Rileggendoli con l'occhio attento al tema della cupidigia si sarebbe rilevato come, finché aveva composto versi nelle mura di Firenze, il poeta non si fosse mai espresso sull'argomento. ${ }^{20} \mathrm{Al}$ contrario, di quel vizio Alighieri si era iniziato a preoccupare solo una volta escluso dalla comunità.

Già nel prosimetro scritto tra il 1306 e il 1308 mentre si aggirava tra la Lunigiana e Lucca, la «cupiditate» appariva per due volte, nel libro IV, come qualifica dell'avidità di ricchezze terrene. Il Convivio in ogni caso fu poco letto e ancor meno commentato durante quegli anni, al contrario del sacrato poema in terzine, dove la "cupidigia” risuonava già dall'Inferno e accompagnava i lettori fino al Paradiso. Segnatamente, nel canto XII del regno infernale la cupidigia veniva deprecata come «cieca». ${ }^{21} \mathrm{E}$ lo stesso nel XXX paradisiaco, dove si incontrata di nuovo una «cieca cupidigia». Qui, per di più, la passione per i beni terreni era presentata quale causa dell'opposizione degli italici all'imperatore Enrico VII: un impulso capace di offuscare l'intendimento («che v'ammalia», Par. XXX, 139), come la cupiditas dell'epistola aveva reso «obcecati» gli scelestissimi fiorentini del 1311. Nell'orizzonte di ricezione fiorentino però le analogie con altri testi dell'autore si limitavano al richiamo di una parola. La cupiditate più o meno ottenebrata che Dante aveva argomentato in lingua madre non appariva né rivolta direttamente contro i Guelfi di Firenze, né connotava specialmente la loro politica. I commentatori cittadini potevano quindi spiegarla con il lessico generale dei peccati morali, in termini di «desiderio d'avere» oppure di «rubare, essere avarizia»,22 come chiosò il divulgatore ufficiale Boccaccio. La cupiditas nella lingua alta dei saperi invece non si poteva tradurre in significati altrettanto generici.

L'esule Alighieri era stato più parsimonioso nell'impiego del termine quando si era espresso con la voce delle dottrine formalizzate, ovvero in discorsi dove la politica fosse inequivocabilmente elevata a materia di riflessione approfondita. Nel catalogo delle scritture dantesche presenti nelle mura fissato dalla Nuova cronica di Villani, solo il trattato imperialista si soffermava sulla cupidigia. E leggendo la Monarchia, fin dalle prime carte dell'opera, la cupiditas trattata dal «filosafo mal grazioso» ${ }^{23}$ - come era ricordato a Firenze - denunciava la sua specifica carica antipolitica, impossibile da scambiare per un peccato cristiano generalizzato come altri.

20 Il censimento delle occorrenze in Pasquini, Cupidigia e Pasquini, Cupidità. Conv. IV, xii. I dati certi sulla composizione del Convivio in Inglese, Vita di Dante, pp. 86-91; sulla diffusione delle cantiche della Commedia, pp. 121-134, 143-147.

21 Inf. XII, 49. Per sacrato poema in seguito, cfr. Casadei, Dante oltre, pp. 35-40, 195-ss.

22 Boccaccio, Esposizioni, cap. 45. Cfr. Barański , «Chiosar», pp. 13-ss.

23 Villani, Nuova cronica, X, 136. 
Nel primo libro del trattato era concentrata la riflessione sul tema, introdotta dalla sentenza per cui laddove la volontà non sia monda da cupidigia, pure la giustizia è spuria. Poco oltre, l'autorità dell'Etica Nicomachea di Aristotele ribadiva l'assunto. Ancora dopo la cupidigia (che deprezza la dimensione umana degli individui) appariva come il contrario della carità (rivolta a fare il bene dei mortali). ${ }^{24} \mathrm{E}$ in entrambi i casi, la giustizia e la carità negate erano di carattere politico. Tutte e due erano considerate indispensabili al medesimo fine: governare rettamente. Quindi, scorsi alcuni paragrafi, si giungeva a una conclusione che valeva da assioma politico, ormai già argomentato in precedenza. «Come mostrato più sopra» - determinava l'autore - solo la monarchia universale era predisposta a una giustizia equa, perché unica istituzione esentata dalla cupidigia politica. L'universalità superiore dell'Impero, per natura, lo liberava dalla necessità di rivendicare frammenti del tutto. Per cui gli eredi dei Cesari erano i soli a potere governare sollevati dall'esigenza di una espansione continua del loro dominio, come invece accadeva «agli altri principi» sparsi per la cristianità. ${ }^{25}$

All'accumulo di ricchezze e alla brama insaziabile di accrescerle, in senso stretto, nell'intera Monarchia non si faceva cenno. La «humana cupiditas» ${ }^{26}$ era invece chiamata in causa un'ultima volta, nella parte conclusiva del trattato, a confermarne le insidie conoscitive e l'irrazionalità politica. Le sue minacce - si ricordava nel terzo libro - insidiavano la facoltà di retta valutazione, riducendo gli uomini in bestie vaganti a briglia sciolta, lanciate sempre in direzione contraria a quanto indicavano sia la ragione dei filosofi che le verità sovrannaturali. La cupidigia insomma confondeva l'orientamento tra le gerarchie degli strumenti di analisi del reale, illudeva di fornire un sapere autonomo, primeggiante e dalle applicazioni senza confini. Effetti non troppo diversi da quelli provocati

24 Mon. I, xi, 6: «nam ubi voluntas ob omni cupiditate sincera non est, etsi assit iustitia»; I, xi, 11: «ad evidentiam primi notandum quod iustitie maxime contrariatur cupiditas, ut innuit Aristotiles in quinto ad Nicomacum». Per la datazione del trattato agli anni della spedizione di Enrico VII, convince la contestualizzazione di Introduzione in Mon. (Quaglioni), pp. XXXVIILVI.

25 «Cum ergo Monarchia nullam cupiditatis occasionem habere possit vel saltem minima inter mortales, ut superios est ostensum, quod ceteris principibus non contigit, et cupiditas ispa sola sit corruptiva iudicii et iustitie preperditiva» (Mon. I, xiii, 7); e poco prima alcuni esempi: «aliis, quorum principatus ad alios terminantur, ut puta regis Castelle ad illum qui regis Aragonum» (Mon. I, xi, 12).

26 «Has igitur conclusiones et media, licet ostensa sint nobis hec ab humana ratione que per phylosophos tota nobis innotuit, hec a Spiritu Sancto (...) qui per coecternum sibi Dei filium Iesum Christum et per eius discipulos supernaturalem veritatem revelavit, humana cupiditas postergaret nisi homines, tanquam equi, sua bestialitate vagantes "in camo et freno" compescerentur in via» (Mon. III, xv, 9). 
dalla «stupefacente cupidigia» ${ }^{27}$ che accecava l'intendimento degli scelestissi$m i$, inficiando la capacità di valutare le proprie condizioni militari e di lasciarsi aperta la via al negoziato. Una cupidigia che induceva all'errore sia circa l'affidamento degli alleati (più indaffarati a difendersi che proteggere altri), sia in merito alla conformazione borghese di Firenze (male apparecchiata alla guerra), o alla tenuta del consenso interno (sotto una imminente pressione esterna), così come impediva di apprezzare tutte le altre evidenze che l'esule esponeva ai reggitori fiorentini nel marzo del 1311.

Anche se non si fosse compulsata la Monarchia del mittente, comunque, la cupiditas iscritta nel messaggio epistolare imponeva di essere decifrata nel medesimo codice interpretativo, cioè come una dinamica dalle ricadute politiche. ${ }^{28}$ Se la materia richiedeva le medesime facoltà intellettuali, la forma però continuava a segnalare che quanto lì esposto andava inteso su tutt'altro piano di realtà. Nel testo dell'Eterni pia providentia infatti la distanza rassicurante che separa il modello teorico dalle sue applicazioni storiche era negata ai lettori. Oltre alla datazione esatta, nella conclusione il chiaro segnale linguistico «hic» cioè «questo divo e trionfante Enrico» ${ }^{29}$ quale personificazione storica dell'autorità imperiale inchiodava la decifrazione dell'intero discorso: la cupidigia, nello specifico, andava connessa a qualcosa che durante gli anni '10 vincolava le scelte politiche degli scelestissimi. Dante non precisò esplicitamente il nesso tra il vizio accecante che affliggeva i Guelfi di Firenze e la tentazione irresistibile verso i nuovi regni di cui pure erano succubi, lasciando che fosse la sovrapposizione tra la logica del suo testo e l'evidenza del contesto di cui i destinatari erano protagonisti a caricare di senso la connessione. In conclusione, proviamo a sciogliere tale intreccio di significati, con la consapevolezza che la decifrazione che a noi richiederà ancora qualche pagina dovette costare pochi istanti ai primi destinatari del messaggio del 1311.

Nel gioco ricettivo su cui si fondava il patto tra autore e lettori di un discorso metaforico come quello dell'epistola, l'identificazione più evidente era quella tra $\mathrm{i}$ «nova regna» e quello del neo sovrano di Napoli, Roberto d'Angiò, che nel 1309 era stato incoronato dalle mani del papa ad Avignone. Fu lui a essere ricordato per oltre un secolo dai fiorentini quale campione italico del fronte antimperiale, argine alla presenza stabile e attiva dell'Impero nella penisola ai tempi di Dante, e in particolare durante la spedizione di Enrico di Lussembur-

27 «O mira cupidine obcecati» (Ep. VI, 12), e si veda anche la sequenza nei paragrafi 12-18. 28 Casadei, Dante oltre, p. 113 identifica un possibile riferimento di Dante al testo dell'epistola nella Monarchia, accolto da Quaglioni in Introduzione, p. XLVII.

29 «Hic divus et triumphator Henricus» (Ep. VI, 25). 
go. ${ }^{30}$ Più in generale, il nipote di Carlo I a Firenze era stimato quale «il più savio re che fosse tra' Cristiani» ${ }^{31}$ nell'ultimo mezzo millennio, ovvero dai tempi di Carlo Magno. Tale valutazione diventò presto una parola d'ordine del guelfismo locale poiché valeva da autolegittimazione: erano state le manifeste virtù di saggezza politica di Roberto ad aver consigliato i regimi cittadini a mantenere sempre saldo un legame specialissimo con il regno, negli interessi della comunità fiorentina. Bene comune e fedeltà angioina erano più che compatibili, e, come in quegli anni sosteneva a Firenze il predicatore domenicano Remigio dei Girolami, una comunità cittadina bene ordinata aveva tutto da guadagnare dalla protezione dei sovrani di Napoli. ${ }^{32}$

Quando Dante scrisse ai concittadini l'angioino era un re nuovo perché da poco incoronato, ma nel decennio precedente si era allenato da protagonista nel mestiere di governare il particolare dominio che gli sarebbe toccato in eredità. ${ }^{33}$ Già da allora la corte di Napoli e gli amici guelfi più solidali con la dinastia avevano preparato la successione, diffondendo un profilo politico del futuro sovrano che rilanciasse l'immagine della monarchia angioina dopo lo sfortunato regno del genitore Carlo II. Rispetto al modulo di una sovranità irruenta e guerriera che caratterizzava la memoria di Carlo I, castigo degli imperatori svevi e fondatore della dinastia di Napoli a fine '200, un cinquantennio dopo si preferì prospettare agli alleati italici una dominazione sovraregionale angioina più elastica e prudente, compendiata nella notoria arte di Roberto nel temporeggiare il più a lungo possibile, lasciare spazi al negoziato, intervenire solo quando indispensabile. ${ }^{34}$

Nell'opinione diffusa, al contrario, la continuità restava salda quanto al peccato tipico della famiglia regia, una tara che ormai appariva trasmessa di padre in figlio assieme al sangue angioino. Simile in questo all'avo conquistato-

30 Ancora il fiorentino Giannozzo Manetti, Vita Dantis, p. 38, sottolineava il protagonismo politico del re di Napoli, poco prima che il poeta avesse inviato «epistulam quandam "ad Florentinos", ut ipse vocat, "intrinsecos" contumeliosam sane scriberet, in qua eos acerbissime insectatur». Ricostruzione degli eventi del 1311 (da un punto di vista dantesco) e rimandi alla bibliografia di riferimento in Somaini, Dante.

31 «Questo re Ruberto fu il più savio re che fosse tra' Cristiani già-ffa cinquecento anni (...) Dolce signore e amorevole fu, e amicissimo del nostro Comune, di tutte le virtù dotato, se non che cominciò a 'nvecchiare l'avarizia il guastava» (Villani, Nuova cronica, XII 10 - ma «iscusavasene per la guerra ch'avea»). Cfr. anche Compagni, Cronica («savio signore e amico de' Fiorentini», p. 141).

32 Boyer, Florence.

33 Su questa fase, rimane indispensabile Caggese, Roberto.

34 Sintesi in Kelly, The New Solomon, da integrare almeno con la bibliografia in Boyer, Roberto. V. ora anche Terenzi, Gli Angiò, pp. 87ess. 
re, anche il savio Roberto aveva dato segni di avarizia fin da principe. ${ }^{35}$ Durante il suo lungo regno il vizio si era acutizzato, e con i decenni era divenuto tanto marcato che neppure i Guelfi fiorentini più ortodossi lo sottacevano, come il filoangioino Villani che lo menzionò nella Nuova cronica (sebbene in modo da completare il monumento di virtù regie con un culmine di umiltà, dal momento che il sovrano era stato il primo a dolersene). La condivisione di tale valutazione nello schieramento guelfo divenne un altro dei luoghi comuni con cui si poteva, a poco rischio, mostrare la propria saggezza politica. Come la consapevolezza esibita delle lotte di parte, della concorrenza tra famiglie, dei danni provocati dall'esclusione politica o del peso degli oneri con cui si finanziava il comune potevano essere denunciati senza danni ideologici, quali mali pressoché inevitabili del sistema di convivenza civile dentro le mura, radicati, costanti e parte dell'identità di lungo periodo della comunità, così anche l'avarizia dei re di Napoli poteva essere rilevata senza mettere in discussione l'ortodossia guelfa dei sostenitori dell'amicizia con gli Angiò. Nessuna contraddizione si frapponeva tra l'avarizia regia e la reputazione morale sia dell'avo Carlo che del discendente Roberto. La loro esistenza terrena era considerata moralmente esemplare, quella di principi attorniati da un apparato di sobrietà severa e circonfuso di pietà. Tutti e due avevano apertamente favorito la spiritualità rigorosa; e il nipote indossava pure il manto di protettore dei frati spirituali seguaci estremi di san Francesco, i più rigoristi circa l'applicazione della povertà cristiana. ${ }^{36}$

$\mathrm{Al}$ contrario dei nefandi peccati aggravati dall'eresia che l'opinione guelfa ancora citavano associati al nome dello svevo Federico II, ultima presenza dell'Impero sul suolo italico dalla metà del '200, la macchia dell'avarizia politica che anche i loro amici politici rilevavano dunque non intaccava le persone della regalità angioina. In altri termini, l'ansia di risparmiare le risorse, nell'assetto dei nuovi regni, valeva quanto una ragione di stato. Come tale, anche il vertice istituzionale ne poteva prendere atto, proprio come i Guelfi fiorentini ricordavano avesse fatto il savio monarca che perpetuavano nella memoria comunitaria. «La guerra»: ${ }^{37}$ così, quanto a lui, il prudente re Roberto giustificava l'economia politica di famiglia davanti agli amici di Firenze.

In breve, avarizia e prudente saggezza del re di Napoli apparivano complementari. Attraverso l'endiadi valoriale si esprimeva la percezione della struttura di un dominio dalla conformazione sfuggente, dai contorni mobili, senza simili

35 L'avarizia degli angioini in Barbero, Il mito angioino, e per Roberto in particolare pp. 124ss. Cfr. Kelly, The New Solomon, pp. 187-188; e, rispetto a Dante, Arnaldi, La maledizione; Del Vento, «L'avara povertà».

36 Kelly, The New Solomon, pp. 74-90.

37 Vedi nota 31 . 
nel passato della penisola. Flessibilità e diramazioni in Provenza, Oltremare, attorno a Roma e negli stati dei papi, oltreché tra signori e comunità fedeli nel regno italico, determinavano una economia politica dell'insieme altrettanto peculiare. ${ }^{38}$ Reggere il dominio angioino comportava, in pratica, una abilità minuziosa nel coordinare le rendite fiscali delle terre direttamente sottoposte alle possibilità di ricavare risorse altrove, sfruttando un circuito di poteri e comunità dalla geografia discontinua e in movimento. ${ }^{39}$

Al tempo della spedizione imperiale di Enrico VII l'avarizia di lungo periodo della casa d’Angiò non doveva certo sorprendere i cittadini eminenti di Firenze. Né tantomeno gli scelestissimi intrinseci, ovvero i Guelfi più convinti dell'adesione alla politica dei «nova regna», ${ }^{40}$ poiché loro conoscevano il sistema meglio di qualsiasi altro gruppo cittadino della penisola. Vi esercitavano infatti parte attiva e indispensabile da molti decenni, da quando cioè gli investimenti politici e finanziari dei loro avi di tre generazioni prima avevano alimentato la sostituzione del controllo svevo imperiale del sud d'Italia con quello dei nuovi sovrani della casata francese. Per amministrare con efficacia e rapidità una economia regia applicata a tempi e spazi strutturalmente sottoposti all'imprevedibilità e alla dispersione, infatti, i re appena giunti avevano prolungato e reso stabile la pratica che li aveva sostenuti durante la conquista. Selezionando i più esperti, affidabili e solventi tra i gruppi mercantili che finanziarono la spedizione del 1266, la dinastia arruolò un personale addetto alle riscossioni fiscali nel regno meridionale ammaestrato da una straordinaria competenza tecnica, acquisita in decenni di esercizi monetari e commerciali, conteggi e spostamenti di risorse, interscambio tra prodotti e denaro contante, coordinamento tra congiunture militari e riscossione di crediti: e tutto ciò su spazi e in proporzioni confacenti a quelle di una confederazioni di domini sparpagliati dalla Sicilia ai confini della Bretagna e della Normandia. Le compagnie dei Mozzi, degli Acciaioli, dei Bardi per prime, seguite rapidamente da Buondelmonti, Scali, Aldobrandini, Visdomini, Peruzzi, Bonaccorsi e uno stuolo sempre crescente di associati (tra cui, in seguito, anche i Villani e Giovanni il cronista), furono così incaricate di maneggiare direttamente le valvole che alimentavano i flussi di denaro del sistema angioino. ${ }^{41}$

38 Abulafia, I regni; Abulafia, L'État angevin; Morelli, Introduzione; Boyer, Conclusions.

39 Boyer, Le fisc; Barbero, L'Italia.

40 Un profilo di gruppo in Najemy, Storia, pp. 86-115. Ancora fondamentale Masi, I banchieri. Per le origini cfr. Jordan, Les origines (e si veda poi Milani, Sulle relazioni).

41 Yver, Le commerce, pp. 289-ss. (circa Villani, pp. 299-300). Ricadute tarde su Firenze in Sapori, La crisi. Si veda anche Poloni, Banchieri; e più complessivo Tognetti, Le compagnie. In prospettiva dantesca, cfr. Cherubini, Dante e le attività. 
I finanzieri di Firenze offrivano un duplice vantaggio ai nuovi regnanti di Napoli, di cui altri stati non potevano godere. Innanzitutto, quei gestori stranieri di prerogative fiscali e pubbliche disponevano in proprio di crediti e risorse autonome, bastevoli a anticipi che non pesassero immediatamente sui sudditi. Un potere determinante per la mobilità continua dell'autorità angioina nei suoi domini sparsi, rischioso da attribuire a un corpo socio-professionale radicato localmente in territori da poco sottomessi. I fiorentini, al contrario, prediligevano reinvestire i profitti in patria e ammassare capitali immobiliari nelle terre attorno al loro comune. La massa di sostanze che alimentava la solvibilità a disposizione dei sovrani, così, gravava altrove. A Firenze i beni di garanzia delle compagnie finanziare sostenevano il peso politico delle grandi famiglie titolari con il loro seguito di associati nel governo cittadino. L'onere di garantire una egemonia territoriale in loco, nel dominio regionale, che tutelasse e accrescesse i capitali accumulati, ricadeva quindi sui reggenti del comune toscano, spingendoli in imprese espansionistiche a danno di comunità e poteri minori ben lontani dal regno, e in fin dei conti marginali negli affari che occupavano la curia regia di Napoli. ${ }^{42}$

Nei primi del Trecento l'implicazione finanziaria e politica con i principi napoletani rappresentava una tradizione ormai identitaria per il gruppo di fiorentini che ne era protagonista, tanto che in molti casi la complicità con l'avarizia angioina era titolo di vanto, strumento di prestigio e distinzione sociale in città. Avanzare denari ai principi si considerava come una pratica che nulla aveva a che spartire con l'avidità peccaminosa di prestatori e usurai privati: semmai poteva essere guardata con il misto di rispetto e ammirazione di cui beneficiavano i maggiori contribuenti al debito pubblico del comune..$^{43}$ Le ricadute dell'amicizia finanziarie con la casa d'Angiò potevano facilmente essere presentate quali benemerenze comunitarie, utili alla sicurezza di tutti i concittadini.

Così era accaduto appena sei mesi prima che l'esule Alighieri inviasse il messaggio epistolare, quando tra il settembre e l'ottobre del 1310 Roberto d'Angiò aveva sostato per tre settimane a Firenze, di ritorno dall'incoronazione avignonese. In quella occasione i governanti cittadini avevano omaggiato i reali con «presenti di grande moneta», ${ }^{44}$ come non si mancò di ricordare nella me-

42 Le ricadute sociali e politiche interne in Raveggi, Tarassi, Medici, Parenti, Ghibellini; e, per il Trecento, in Najemy, Storia, pp. 117-151. Sul sistema invece cfr. Petralia, I Toscani e Petralia, Fiscalità (ma cfr. anche l'intero volume Lo stato territoriale).

43 Cfr. Todeschini, La banca.

44 «Albergò in casa de’ Peruzzi dal Parlagio, e da' Fiorentini gli fu fatto grande onore, e armeggiata, e presenti grandi di moneta» (Villani, Nuova cronica, X, 8); il sovrano fu ospite di Giotto di Arnoldo Peruzzi (cfr. Yver, Le commerce, pp. 302-ss). 
morialistica locale. Il dono meritava di essere evidenziato nelle cerimonie di accoglienza perché rappresentava anche un segnale politico. All'approssimarsi della spedizione imperiale nella penisola, con quel pagamento esibito il comune riattivava in pubblico la compartecipazione al sistema di dominio guelfo rappresentato dal nuovo sovrano. In particolare, la spontaneità dell'offerta confortava davanti alla cittadinanza la libertà delle comunità, più che suggerire un rapporto di sottomissione. Per gli osservatori più savi, per i cittadini più informati circa le connessioni tra diverse dinamiche di poteri e interessi in campo quei gesti ufficiali inoltre formalizzavano nel linguaggio delle istituzioni locali una strategia su scala sovraregionale, messa in atto già da mesi. Per gli scelestissimi soprattutto servivano a ratificare attraverso un contatto personale l'impegnativo patto di fedeltà finanziaria che aveva sorretto il passaggio successorio ai vertici del regno.

L'ingresso nelle mura fiorentine di Roberto e della corte nel settembre 1309 aveva segnato l'ultima tappa rilevante di un viaggio di incoronazione iniziato dalla capitale del regno, nella primavera dell'anno precedente, che era valso da presa di possesso del circuito angioino. Attraverso la Provenza e poi, lentamente, ridiscendendo nella penisola via terra, dal nord-ovest, il corteo aveva sostato tra le maggiori comunità guelfe o a vario titolo amiche dei re di Napoli. ${ }^{45}$ Non a caso, in quei mesi di confronto tra le due sovranità sul suolo italico l'immagine politica e letteraria del copricapo regio apparve particolarmente appropriata per rappresentare lo scontro in atto. ${ }^{46}$ Precedente di qualche settimana l'arrivo di un aspirante imperatore che cingeva solo una corona germanica, la parata itinerante del sovrano appena consacrato dal pontefice rese generalizzata tra $i$ cittadini italici, e urgente, la questione che l'esule Alighieri avrebbe inviato ai reggitori di Firenze pochi mesi dopo: nella penisola la presenza imperiale determinava l'esistenza di un solo regnum, la cui corona doveva essere cinta in Lombardia da ciascun re dei romani eletto all'Impero? Oppure a Napoli, e poi in Avignone, si era rinnovata l'esistenza di una alternativa collaudata, durevole, che si rigenerava con la prevedibilità della successione per sangue, in grado di seguire ritmi e forme più efficaci nel dare ordine all'insieme italico?

Gli scelestissimi cui si rivolgeva Dante nel marzo 1311 non avevano atteso la celebrazione pubblica dell'amicizia angioina a Firenze di sei mesi prima per rispondere con i fatti. Fin da prima che il sovrano e la corte salpassero sulla rotta di Provenza, a Napoli la burocrazia regia aveva registrato l'apertura di un credito presso le compagnie di Bardi e Peruzzi con cui saldare ogni necessità,

45 Il percorso si ricostruisce da Caggese, Roberto, pp. 102-ss.

46 Brilli, Fontes Baratto, Montefusco, Sedurre l'imperatore; più in generale, cfr. Zug Tucci, Henricus. 
in ciascuna tappa della trasferta sovraregionale. Tra censi dovuti alla sede apostolica, sostegno alla beatificazione del santo di famiglia, sussidi ai reggenti nel Mezzogiorno, spese di apparato e di milizie, quando nove mesi dopo lasciò Firenze per rientrare nella sua capitale, Roberto riconosceva volentieri agli scelestissimi nelle mura debiti per circa mezzo milione di fiorini. ${ }^{47}$

Nelle circostanze in cui i governanti di Firenze ricevettero l'epistola datata al marzo 1311, la cupiditas ottenebrante denunciata dall'esule attivava codici di decifrazione e suggeriva significati piuttosto evidenti ai lettori. Ciò che impediva la corretta visione della geopolitica italica da dentro le mura - per come si esprimeva il messaggio - andava riconosciuto in una commistione tra uso delle istituzioni comunitarie e finanza, tipica del guelfismo locale. Una pratica da non confondere con una banale brama di ricchezze. Sarebbe stato fare un insulto alle capacità intellettuali dei destinatari, ovvero una contraddizione incomprensibile in un retore rinomato quanto Dante, che per gli scelestissimi mobilitava il linguaggio dei saperi alti. La cupidigia di cui erano adepti gli interpellati equivaleva a una saggezza politica diffusa, che l'autore contestava come fallace. La ragione apparentemente neutra che si fondava sul calcolo matematico e la straordinaria possibilità di controllare le dinamiche di forza con movimenti invisibili di risorse monetarie - spiegava il messaggio di Dante - era degenerata in una ideologia imperante che sfuggiva ai suoi stessi sostenitori, accecandoli sulle possibilità di modulare diversamente la loro identità di savi mercanti e cittadini.

La cupidigia degli scelestissimi, tuttavia, non andava neppure liquidata come una versione comunale della tendenza espansiva di ogni potere particolare, propria a quegli «altri principi» ${ }^{48}$ della cristianità criticati nella Monarchia come occupanti abusivi delle spettanze imperiali. Nel caso in questione, all'avarizia politica dei capetingi di Napoli si poteva concedere l'indulgenza dovuta a chi aspira a conservarsi nel tempo. Necessaria per governare su qualsiasi dimensione di stato, l'oculatezza nell'impiego delle risorse si accordava alle virtù utili alla durata e alla prosperità di famiglia, sia borghesi che principesche, come insegnavano la saggezza da conversari in piazza e pure le dottrine aristoteliche. La passione oscurante che animava i concittadini dell'esule nell'Eterni pia providentia, inversamente, appariva rivolta all'esterno della comunità, oltre le mura e il contado. Non mirava a conservare bensì ad accrescere, senza limiti di spazi e confini, coinvolgendo persino altri stati come il regno di Roberto d’Angiò.

47 Baso le stime sui dati di Yver, Le commerce, pp. 297-307, che converto da once in fiorini (circa le proporzioni: nel 1341, e a un prezzo non di favore, il comune acquistò la città di Lucca per 250,000 fiorini).

48 Cfr. supra, nota 25. 
Guelfi del regime che sostenevano la monarchia degli Angiò interpretavano la libertà di approfittare delle debolezze di un sistema politico artificioso quale garanzia di indipendenza, dentro e fuori le mura. I più coscienziosi e savi ponderassero pure quanto i vantaggi finanziari dei circuiti mercantili fossero reimpiegabili per la tutela della libertas di Firenze, acquistando terre, pagando difese e alleanze, ostacolando le minacce con l'arma vischiosa del denaro. In quelle condizioni, la comunità era condannata comunque a un «ergastolo di vera servitù» - su questo allertava il messaggio di Dante ai concittadini -, la stessa di cui resta prigioniero chiunque fondi la propria fortuna sui debiti altrui. 
